\title{
Factors Affecting Maternal Health Care Services Utilization in Nepal: Insight from the Nepal Demographic Health Survey 2006 and 2011
}

\author{
Gauri Shrestha
}

Submitted: 21 June 2017; Accepted: 23 July 2017

\begin{abstract}
Background: In this study, a maternal health care service is analyzed under the components of place of delivery (POD). POD is highlighted as 'home delivery' and 'institutional delivery'.

Objective: The objective of this study is to analyze factors associated with the utilization of health institute as delivery and estimate the probabilities of institutional delivery using some selected independent variables.

Materials and Methods: Data used were extracted from individual recode of a data file of Nepal Demography Health Survey (NDHS) 2006 and 2011. The unit of analysis for this study is Ever Married Women (EMW) who had at least one live birth in the five years preceding the survey. Sample of this study consists of 4182 EMW for 2006 NDHS and 4079 EMW for 2011 NDHS. The dependent variable is place of delivery. The independent variables are mixture of categorical and continuous variables. For building of suitable statistical models, various types of models were explored and different measure of models adequacy test were applied. Finally, two logistic regression models were developed separately for 2006 and 2011 NDHS data.

Results: Fitted model showed several variables: education level, wealth index, birth order, residence and ANC by provider were highly significant predictors for the selection of place of delivery for 2006 NDHS but in 2011 NDHS age was also highly significant. Goodness of fit tests (Hosmer-Lemeshow chi-square statistic), multicollinearity diagnostics, residual analysis and outliers (leverage value) showed that both models fit well to the proposed logistic regression model.

Conclusion: A Comparative assessment of model coefficients between 2006 and 2011 NDHS, it is found that the values are only slightly different for most of the predictors under consideration demonstrating consistency of associations found in the two surveys.
\end{abstract}

Keywords: Ever married women, institutional delivery, logistic regression model, multicollinearity.

Address correspondence to the author: Central Department of Statistics, Tribhuvan University, Kirtipur, Kathmandu, Nepal Email: gaurishrestha@yahoo.com 


\section{INTRODUCTION}

Maternal health care is a crucial part of any health care system. Health care that a woman receives during pregnancy, at the time of child birth and soon after, is important for the survival and well-being of mother and the new born. Utilization of maternal health services is a complex behavioral phenomenon. Empirical studies of preventive and curative services have often found that use of health services is related to the availability, quality and cost of the services, that will undoubtedly influence on individual's decision. Other factors, such as the social structure, health belief and personal characteristics of the users and the community are also determining factors (Anderson \& Newman, 1973; Kroeger, 1983; Becker et al, 1993; Sarin, 1997). Use of maternal health care services is an effective approach to reducing the risk of maternal morbidity and mortality.

Reports show that more maternal deaths are due to absence of adequate number of place of delivery (POD) and skilled assistance. So, regarding the maternal health care services, this study focuses on the importance of a POD as maternal health care services. The objective of delivery at a health institute is to protect the life and health of the mother and her child by ensuring safe and hygienic condition during deliveries. In this study, POD is highlighted as 'home delivery' and 'institutional delivery'. Institutional delivery meaning delivery in health institute which includes all type of facilities in government/ private hospitals/hospital running by NGO's/clinics/ centers or other health institutions. Delivery at home includes respondent's home or others (non-health institutions).

According to official data, the ratio for 1990 to $1996 \mathrm{~s}$, the MMR stood at around 539 deaths per 100,000 live births (WHO, 1996). It slowly decreased to 415 in 2000 (Nepal Planning Commission [NPC], 2002). The most recent available figure is the Nepal Demographic and Health Survey (NDHS) estimation of 281 per 100,000 live births in 2006 (Ministry of Health and Population (MOHP) [Nepal], New ERA, and Macro International Inc., 2007). Percentage of births taking place in a health institute has more than doubled in last five years (9 percent in 2001, 18 percent in 2006 and 39 percent in 2011). Even though the rate of birth taking place in a health institute has increased, still three out of five (61 percent) births take place at home (Ministry of Health [Nepal], New ERA, and ORC Macro, 2002; MOHP, 2007; Ministry of Health and Population (MOHP) [Nepal], New ERA, and ICF International Inc., 2012). This fact is a serious obstacle to reduce maternal mortality as women get less facility at home than in a health facility.

There has been considerable debate in the literature as to whether the mere provision of health services will lead to increase utilization? (Magadi, Madise and Rodrigues, 2000; Obermeyer, 1993; Becker, Peters, Gray, Gultiano and Blake, 1993). Provision of services may not be enough, as people 
will not make use of the available services if they do not have the perceived need to use them. Therefore, it has been argued that utilization of health services is affected not only by access but also by demand for services. People must be made aware of the importance and benefits of using such kind of services. Hence even after controlling for the availability of resources, some women are more likely to use maternal health services than others. This suggests that characteristics of health services may not be the only explanatory factors. Therefore the present study will examine the impact of socio-economic and demographic characteristics to explain why some women are more likely to use maternal health services during pregnancy while others are not.

Review of the papers pertaining to the factors with use of MHCS utilization, logistic regression model has been frequently applied. Some examples are evident from paper published by Elo (1992); Becker et al. (1993); Matsmura and Gubhaju (2001); Latemo (2003); Thind, Mohini and Banarjee (2008); Adeleke and Adepoju (2010); and Shrestha and Shrestha (2011). Many studies reported that increased income positively affects utilization of health care services (Elo, 1992; Chakraborty, Islam, Chowdhury, Bari and Akhter, 2003). Women from poor wealth index may have difficulty in paying for household cost and are less likely to use maternal health services. (Gabrysch and Campbell, 2009) Studies also indicate that women, whose husband has higher status occupation, are more likely to use maternal health services. This is because such occupation is usually associated with greater wealth, making it easier to bear the cost of health care. A study in Nepal has shown that women are less likely to use maternal health services, when they do not have personal control over finance (Gage and Calixte, 2006; Fruta and Salways, 2006). It suggests that an interaction between autonomy and family wealth index produces maternal health service utilization.

This study has made an effort to identify the predictors of place of delivery (health institute/ home) by developing a logistic regression model. Predicted probabilities for delivery at health institute, another logistic regression was fitted with some important variables (highest education level, wealth index and place of residence). It is displayed by graph. Besides, to get a better understanding of why women do not give birth at a health institute, reason of not delivery in health institute is also analyzed.

\section{MATERIALS AND METHODS}

\section{Data}

The data for this study is chosen from the Demographic Health Survey (DHS), 2006 and 2011 of Nepal. The 2006 NDHS and 2011 NDHS are third and fourth comprehensive survey conducted in Nepal as part of the world wide DHS project. The unit of analysis for both studies is Ever Married 
Women (EMW) who had at least one live birth in the five year preceding the survey. For those EMW, who had more than one birth, only utilization behavior of maternal health services associated with most recent pregnancy within five years was considered, so the sample of this study consists of 4182 EMW for 2006 NDHS and 4079 EMW for 2011 NDHS.

Details of the survey procedure and sampling design are available in the individual survey report.

\section{Variables}

Dependent variable was created from questionnaires included in the maternal health component of the DHS questionnaire. As noted in the literature review, majority of maternal deaths and disabilities occur around the time of delivery. For this reason, delivery care represented by place of delivery was selected as dependent variable. The place of delivery was collapsed to create a dichotomous variable on the basis of whether the woman delivery at health institution or home. The health institution includes all type of facilities in government/ private hospitals/hospital running by NGO's/clinics/ centers or other health institutions. Delivery at home includes respondent's home or others (non-health institutions). A number of independent variables are taken into account based on scientific literature review. The independent variables are mixture of categorical and continuous variables. These variables are current age, education level (no education/ Primary/ secondary and higher), Birth in last five years (1/2/more than 2), wealth index (poorest/poorer/middle/richer/richest), Occupation (never work/agriculture sector/ modern sector), residence (rural/urban), ANC by provider (by no one/by informal sources/by SBA), religion (Hindu/others) and sex of household head (male/female).

\section{Data analysis}

Descriptive analysis is performed to observe the difference in delivery at health institute with respect to different variables, which are likely to be associated with it. For building of suitable statistical models, various types of models were explored and different measure of models adequacy test were applied. Finally, two logistic regression models were developed separately for 2006 and 2011 NDHS data to assess the influence of predictors on use of place of delivery.

Let $Y_{i}=\left\{\begin{array}{c}1 \text { if the delivery is at health institute } \\ 0 \text {, otherwise }\end{array}\right.$

Logistic regression extended to model with multiple explanatory variables $(X)$. The model for $\pi_{i}=$ $\mathrm{P}\left(Y_{i}=1\right)$ probability that $Y_{i}=1$ and $1-\pi_{\mathrm{i}}=\mathrm{P}\left(Y_{i}=0\right)$ probability that $Y_{i}=0$ at values $X=\left(X_{1}, X_{2}, \ldots X_{k}\right)$ of $k$ predictors and the subscript $i$ denote $i^{\text {th }}$ individual and the model assumed is

$$
\pi_{i}=\frac{1}{1+e^{-\left(\beta_{0}+\sum_{j=1}^{k} \beta_{j} X_{j}\right)}}
$$


and

$$
1-\pi_{i}=\frac{e^{-\left(\beta_{0}+\sum_{j=1}^{k} \beta_{j} X_{j}\right)}}{\left.1+e^{-\left(\beta_{0}+\sum_{j=1}^{k} \beta_{j} X_{j}\right.}\right)}
$$

Therefore,

$$
\frac{\pi_{i}}{1-\pi_{i}}=e^{\beta_{0}+\sum_{j=1}^{k} \beta_{j} X_{j}}
$$

Then logit or log odds of having $Y_{i}=1$ is modeled as a linear function of the explanatory variables, that is

$$
\ln \frac{\pi_{i}}{1-\pi_{i}}=\beta_{0}+\sum_{j=1}^{k} \beta_{j} X_{i j}
$$

where $X_{j},(\mathrm{j}=1,2, \ldots \mathrm{k})$ are explanatory variables. $0 \leq \pi_{i} \leq 1, \mathrm{j}=0,1,2, \mathrm{k}$, and $\mathrm{i}=1,2 \ldots \mathrm{n}$.

Using the method of calculus, a set of values for $\beta_{0}$ and the $\beta_{j}$ can be calculated that maximize $\mathrm{L}$ and the resulting values are known as maximum likelihood estimates (MLE's). A well-known NewtonRaphson iterative method is used to solve the equations, which is known as iteratively weighted least square algorithm. In brief, this iterative procedure for maximizing $\mathrm{L}(\beta)$ works with a linear approximation of the derivative of $\mathrm{L}(\beta)$ with respect to $\beta_{0}, \beta_{1}, \ldots, \beta_{k}$ and an initial estimate of $\beta_{0}, \beta_{1}, \ldots$, $\beta_{k}$ is obtained. From there an updated estimate of $\beta_{0}, \beta_{1}, \ldots, \beta_{k}$ is obtained. Iteration continues until a convergence criterion is reached.

\section{Model adequacy test}

Several standard measures of model adequacy tests have been considered in order to examine how well the fitted models match the observed data. The overall fit of the model is assessed using the $\log$ likelihood (LL) statistics. The Hosmer-Lemeshow goodness of fit statistics (Hosmer \& Lemeshow, 2000) proposes a Pearson's statistic to compare the observed and fitted counts for the partition. To support the model, a significance value greater than .05 is needed that is if $p$-value for the Hosmer \& Lemeshow goodness of fit test is greater than 0.05 , we will not reject the null hypothesis that there is no difference between observed and model predicted values, implying that the model estimates are adequate to fit the data at an acceptable level. The statistical significance of the estimated coefficients is done via Wald statistic (that is, to test the null hypothesis in logistic regression that a particular logit (effect) coefficient is zero). If the coefficient is significantly different from zero then we can assume that the predictor is making significant contribution to the prediction of outcome (Y).

As logistic regression is not a linear model, we can't calculate $\mathrm{R}^{2}$ directly as for linear regression model. A large pseudo $R^{2}$ indicate that more of the variation is explained by the model, from a 
minimum of 0 to maximum of 1 . It should also be noted that pseudo $R^{2}$ values tend to be very low for logistic regression model, much lower than for linear regression model. This is because we are trying to predict the outcome whereas the model only given us the probability of outcomes. Residual analysis for logistic regression is complicated by the fact that the errors are not normally distributed. The deviance residuals provide information about the fit of individual cases, or more to the point, the contribution of a case to the (overall) deviance. Residual should be checked for outliers and influential observations. Leverage statistics should lie between 0(the case has no influence whatsoever) and 1(the case exerts complete influence over the model), tell us about whether certain cases are welding undue influence over model. Influential values are points that have exerted excessive influence on the regression coefficient estimates. An influential point affects the statistical significance as well as the strength and direction of the association between a response variable and predictor variables. In this study to detect outliers and influential cases that have a substantial impact on the fitted logistic regression model, appropriate graphs were used. Lastly, multicollinearity is assessed by computation of Variance Inflation Factor (VIF).

\section{RESULTS}

\section{Descriptive analysis}

Some descriptive analysis are performed to examine the difference in place of delivery with respect to different relevant variables which can be linked to POD and changed in POD observed in 2011 NDHS compared to 2006 NDHS. Table 1 showed that the proportion of delivery at health institute increased from 19 percent in 2006 to 40 percent in 2011 even though 60 percent of women gave birth in home.

Table 1. Distribution of use of POD in NDHS 2006 and 2011,

\begin{tabular}{llrrcc}
\hline Variables & Description & \multicolumn{2}{c}{2006} & \multicolumn{2}{c}{2011} \\
\cline { 3 - 6 } & & Frequency & Percent & Frequency & Percent \\
\hline Place of & At home & 3390 & 81.1 & 2464 & 60.4 \\
delivery & At health institute & 792 & 18.9 & 1615 & 39.6 \\
\hline
\end{tabular}

The mean age has remained almost same (27 years) in both surveys. More than 70 percent of women have one birth in last five years, which was higher than 2006 survey (66 percent). The secondary/ higher educated women were higher in 2011 NDHS (36 percent) as compared to 2006 NDHS (24 percent). It can be seen in table 2 that 64 percent of the women's occupation were agriculture in 2011 as compared to 74 percent from 2006 NDHS. Large gaps exist in education level of women between the two surveys, it is reported that approximately 36 percent of women have higher and secondary education level in 2011 compared to 24 percent of women in 2006. 
Table 2. Socio- economic and demographic characteristics in NDHS 2006 and 2011.

\begin{tabular}{|c|c|c|c|c|c|}
\hline \multirow[t]{2}{*}{ Variables } & \multirow[t]{2}{*}{ Description } & \multicolumn{2}{|c|}{2006} & \multicolumn{2}{|c|}{2011} \\
\hline & & Freq. & percent & Freq. & percent \\
\hline \multirow[t]{2}{*}{ Age } & Age of respondent & Mean $=$ & Min=15 & Mean $=$ & $\operatorname{Min}=15$ \\
\hline & & 27.04 & $\operatorname{Max}=49$ & 27.0 & $\operatorname{Max}=49$ \\
\hline Education & No education & 2455 & 58.7 & 1765 & 43.3 \\
\hline \multirow[t]{2}{*}{ Level } & Primary & 745 & 17.8 & 817 & 20.0 \\
\hline & Secondary or higher & 982 & 23.5 & 1495 & 36.7 \\
\hline \multirow[t]{5}{*}{ Wealth index } & Poorest & 1111 & 26.6 & 1160 & 28.4 \\
\hline & Poorer & 866 & 20.7 & 832 & 20.4 \\
\hline & Middle & 751 & 18 & 739 & 18.1 \\
\hline & Richer & 773 & 18.5 & 677 & 16.6 \\
\hline & Richest & 681 & 16.3 & 671 & 16.5 \\
\hline No. of births & One & 2740 & 65.5 & 2972 & 72.9 \\
\hline in last five & Two & 1287 & 30.8 & 992 & 24.3 \\
\hline years & Three or more & 155 & 3.7 & 115 & 2.8 \\
\hline \multirow[t]{3}{*}{ Occupation } & Never work & 750 & 17.9 & 962 & 23.6 \\
\hline & Agricultural sector & 3099 & 74.1 & 2603 & 63.8 \\
\hline & Modern sector & 333 & 8.0 & 514 & 12.6 \\
\hline ANC by & By none & 1161 & 27.8 & 611 & 15.0 \\
\hline \multirow[t]{2}{*}{ provider } & By informal sources & 1071 & 25.6 & 1079 & 26.5 \\
\hline & By SBA & 1950 & 44.6 & 2389 & 58.5 \\
\hline \multirow[t]{2}{*}{ Residence } & Urban & 995 & 23.79 & 897 & 21.99 \\
\hline & Rural & 3187 & 76.21 & 3182 & 78.01 \\
\hline \multirow[t]{2}{*}{ Religion } & Hindu & 3633 & 86.9 & 3490 & 85.3 \\
\hline & Others & 549 & 13.1 & 599 & 14.7 \\
\hline Sex of & Male & 3294 & 78.8 & 3002 & 73.6 \\
\hline household & Female & 888 & 21.2 & 1077 & 26.4 \\
\hline Total & & 4182 & 100 & 4079 & 100 \\
\hline
\end{tabular}

Table 3 showed the chi square test of POD by some independent variables. Most of the variables are found statistically significant in explaining the delivery at health institute for both 2006 and 2011 NDHS. Sex of the household head is found to be statistically not significant in both surveys. Religion is not significant for 2006 NDHS but significant for 2011 NDHS. Bivariate analysis of predictors and POD is not shown here. 
Table 3. Chi square test of POD by independent variables in NDHS 2006 and 2011.

\begin{tabular}{lcc}
\hline \multirow{2}{*}{ Variables } & \multicolumn{1}{c}{2006} & 2011 \\
\cline { 2 - 3 } Education Level & $\chi^{2}$ value $(p$ value $)$ & $\chi^{2}$ value $(p$ value $)$ \\
Wealth index & $705.59(<0.001)$ & $662.44(<0.001)$ \\
Birth in last five years & $874.59(<0.001)$ & $946.20(<0.001)$ \\
Occupation & $92.11(<0.001)$ & $67.48(<0.001)$ \\
ANC by provider & $456.93(<0.001)$ & $546.95(<0.001)$ \\
Residence & $<0.001(627.89)$ & $694.39(<0.001)$ \\
Religion & $<0.001(429.36)$ & $409.69(<0.001)$ \\
Sex of household head & $0.728(0.121)$ & $13.19(<0.001)$ \\
\hline
\end{tabular}

\section{Statistical modeling}

Forward stepwise procedure is used to determine which of the predictors should be included in the model. First step in the process is usually to assess the significance of predictors in the model. This usually involves formulation and testing of statistical hypotheses to determine whether the independent variables in the model are significantly associated to the response variable. The change in deviance follows a chi-square distribution with 13 (14 for 2011 NDHS) degree of freedom and measures how well the independent variable affect the outcome or response variable. In this study chi square $=1248.316(1258$ for 2011 NDHS $)$ with $p$ value $<0.001$. This is a test of null hypothesis that adding the predictors to the model has not significantly increased our ability to predict the delivery at health institute. Hence it indicated that, we reject the null hypothesis and we may infer that at least one and perhaps most of the coefficients are different from zero and we may infer as a whole the predictors have significant contribution to predict the response variable. The overall fit of the model is assessed using the log likelihood (LL) statistics. In this analysis rather than reporting log likelihood itself, the value is multiplied by -2 (-2LL, which has an approximately chi square distribution). At the final stage -2LL should be less than the value when only constant is included in the model (lower value of -2LL indicates that the model is predicated the outcome variable more accurately). When only constant was included, -2LL=4059.276 (5476.686 in 2011 NDHS) but when other variables have been included, this -2LL has been reduced to 2810.96 (3950.213 in 2011 NDHS). This reduction tells us that the model is better predicting output variable than it has only the constant included. In the above fitted models, the $\mathrm{p}$ values are highly significant so we can say that over all model is predicting POD significantly better than it was only constant included. 


\section{Model parameter estimates and their interpretations}

Table 5 showed the estimated coefficients, odds ratios and the 95 percent confidence interval. Estimated models showed that most of the parameter coefficients are statistically significant with $\mathrm{p}$ value less than 0.001. Education level, residence, wealth index and ANC by provider are positively associated whereas birth in last five years and occupations are negatively associated with the dependent variable in both model. Estimated model coefficients and corresponding values of odds ratios revealed the following important results on delivery at health institute when values of predictor variables are changed from one level to another.

- Increase in age of women decreases the delivery at health institute with OR 0.965 which means that increase in 1 year of age reduce the delivery at health institute by 3.5 percent Nepalese married women based on 2011 NDHS data.

- Urban women showed more delivery at health institute compared to rural women with OR 1.603 which can be understood as women living in urban areas 1.604 times more to delivery at health institute compared to rural women based upon 2006 NDHS data whereas the value is higher based upon 2011 NDHS data (OR 1.804).

- Education, again, have significantly strong positive associations with the use health institute as place of delivery in both survey. Women with secondary/higher education level are almost 2.29 times more to delivery at health institute than odds of women with no education in 2011 NDHS data whereas the value is higher based upon 2006 NDHS data (OR 3.29).

- As compared to poorest women, the odds ratio of poorer/ middle/ richer/ richest women have more odds ratios as shown in table 4. All values are highly significant compared to $2006 \mathrm{NDH}$ $(\mathrm{p}<0.05$ for poorer, $\mathrm{p}<0.001$ for middle richer and richest). The odd ratios are also increased compared to 2006 NDHS data. This suggests that as the economic condition of Nepalese women improve the odds of having delivery at health institute increases gradually.

- Interestingly, the relationship between women's occupation and place of delivery has an inverse association. Odds ratios of women whose work is in agricultural sector are found to be lower (0.548 and 0.555 for 2006 and 2011 NDHS respectively) which implies that 45 percent less likely to give birth at health institute compare to women who never work. Although not statistically significant, it is suppressing that women whose occupation is in modern sector are 7 percent less likely to delivery at health institute as compare to women whose occupation is never work in both survey. Women who never work may be associated with household of high economic status and for this reason, an inverse may have been found. As compared to women with ANC by no one, the odds ratio of ANC by informal sources and by SBA women have more odds ratios as shown in table 4 in both survey. The odds ratios indicates that the women having 
antenatal care by SBA are likely to have 7.07 times higher to delivery at health institute compare to women having ANC by no one which is higher than 2006 NDHS (5.77).

Table 4. Model coefficients and odds ratios in NDHS 2006 and 2011.

\begin{tabular}{|c|c|c|c|c|c|c|}
\hline \multirow{4}{*}{$\begin{array}{l}\text { Explanatory } \\
\text { variables }\end{array}$} & \multicolumn{3}{|c|}{2006} & \multicolumn{3}{|c|}{2011} \\
\hline & Estimated & Odds & 95\% Wald CI & Estimated & Odds & $95 \%$ Wald CI \\
\hline & Coefficient & Ratio & for $\mathrm{OR}$ & Coefficient & Ratio & for $\mathrm{OR}$ \\
\hline & $(\beta)$ & $\left(\mathrm{e}^{\beta}\right)$ & Lower- Upper & $(\beta)$ & $\left(\mathrm{e}^{\beta}\right)$ & Lower- Upper \\
\hline Constant & $-3.355^{* *}$ & 0.035 & & $-1.07^{* *}$ & 0.191 & \\
\hline Age & - & - & - & $0.036^{* *}$ & 0.965 & $0.950-0.980$ \\
\hline \multicolumn{7}{|l|}{ Residence } \\
\hline Rural (R) & - & - & - & - & - & - \\
\hline Urban & $0.472^{* * *}$ & 1.603 & $1.292-1.988$ & $0.590^{* * *}$ & 1.804 & $1.474-2.208$ \\
\hline \multicolumn{7}{|l|}{ Education level } \\
\hline No education (R) & - & - & - & - & - & - \\
\hline Primary & $0.305^{*}$ & 1.356 & $1.040-1.768$ & $0.391^{* *}$ & 1.478 & $1.195-1.857$ \\
\hline Secondary/higher & $1.175^{* *}$ & 3.239 & $2.576-4.047$ & $0.829^{* * *}$ & 2.290 & $1.865-2.807$ \\
\hline \multicolumn{7}{|l|}{ Wealth index } \\
\hline Poorest (R) & - & - & - & - & - & - \\
\hline Poorer & 0.337 & 1.400 & $0.951-2.061$ & $0.299^{*}$ & 1.348 & $1.057-1.720$ \\
\hline Middle & $0.395^{*}$ & 1.484 & $1.010-2.181$ & $0.533^{* * *}$ & 1.704 & $1.331-2.181$ \\
\hline Richer & $0.626^{* *}$ & 1.869 & $1.294-2.700$ & $0.718^{* * *}$ & 2.051 & $1.564-2.690$ \\
\hline Richest & $1.294^{* *}$ & 3.647 & 2.467- 5.392 & $1.516^{* *}$ & 4.555 & $3.272-6.339$ \\
\hline \multicolumn{7}{|l|}{ Birth in last 5} \\
\hline years & - & - & - & - & - & - \\
\hline $1(\mathrm{R})$ & $-0.610^{*}$ & 0.543 & $0.433-0.682$ & $-0.216^{*}$ & 0.806 & $0.669-0.0971$ \\
\hline 2 or more & $-0.869^{*}$ & 0.420 & $0.209-0.843$ & -0.514 & 0.598 & $0.350-1.213$ \\
\hline \multicolumn{7}{|l|}{3 or more } \\
\hline \multicolumn{7}{|l|}{ Occupation } \\
\hline Never work (R) & - & - & - & - & - & - \\
\hline Agricultural & $-0.602^{* *}$ & 0.548 & $0.433-0.694$ & $-0.588^{*}$ & 0.555 & $0.454-0.679$ \\
\hline sector & -0.057 & 0.945 & $0.692-1.291$ & -0.070 & 0.932 & $0.716-1.213$ \\
\hline \multicolumn{7}{|l|}{ Modern sector } \\
\hline \multicolumn{7}{|l|}{ ANC by provider } \\
\hline No one (R) & - & - & - & - & - & - \\
\hline Informal sources & $0.572^{* *}$ & 1.771 & $1.159-2.708$ & $0.965^{* *}$ & 2.624 & $1.816-3.791$ \\
\hline SBA & $1.753^{* *}$ & 5.769 & $4.002-8.318$ & $1.956^{* *}$ & 7.068 & $5.003-9.985$ \\
\hline
\end{tabular}




\section{Model adequacy test}

- For goodness of fit, Hosmer-Lemeshow chi-square statistic with 8 degree of freedom is 6.243 with $\mathrm{p}$ value 0.620 (7.901 with $\mathrm{p}$ value 0.443 for 2011 NDHS). The large $p$ value signifies that there is no difference between the observed and predicted values, implying that the model fits the data at an acceptable level.

- Negelkerke $R^{2}$ (pseudo $R^{2}$ ) measure the proportion of the variation in the dependent variable that can be explained by predictors in the model. Here $R_{N}^{2}=0.415$ and 0.423 for 2006 and 2011 NDHS respectively. It should also be noted that pseudo $\mathrm{R}^{2}$ values tend to be very low for logistic regression model, much lower than for linear regression model. This is because we are trying to predict the outcome where as the model only given us the probability of outcomes.

- To detect outliers and influential cases those have a substantial impact on the fitted logistic regression model, there are no high values of DFBETAS (all values less than 0.025) which means that there are no influential observations for the individual regression coefficients.

- Residual analysis was carried out via various graphs. A check of the standardized residuals for the place of delivery is presented in figure1. It revealed that all have values less than absolute value of two indicating the absence of outliers in the model implying the models are adequate.

(a)

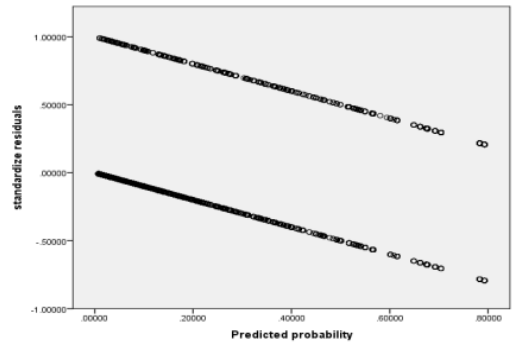

Fig.1. Scatterplots for outliers (standardize residuals), (a) NDHS 2006, (b) NDHS 2011.

Another method of detecting outliers is leverage value. From the scatter plots of leverage values for the place of delivery shown in figure 2, leverage values are less than one indicating the absence of outlying observation. 
(a)
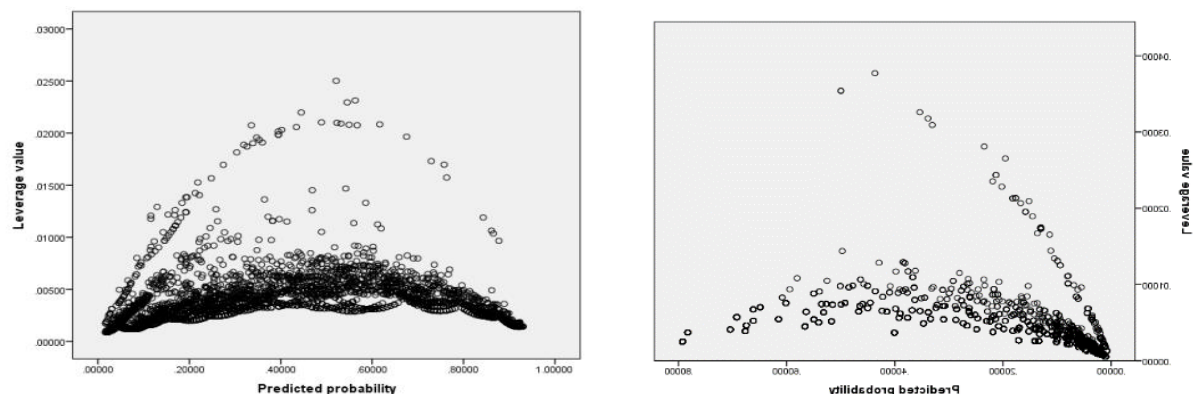

(b)

Fig. 2. Scatter plots for diagnostic checking (Leverage Value), (a) NDHS 2006, (b) NDHS 2011.

In addition, Cook's distance is proposed to measure the effect of excluding any specific observation on the set of parameter estimates. Cook (1977) gives the value of $D, d>1$ identifies cases that might be influential. Figure 3 indicated that there are no large values of Cook's distance $(\mathrm{Di}<1)$, means that there are no influential cases having an effect on the model.

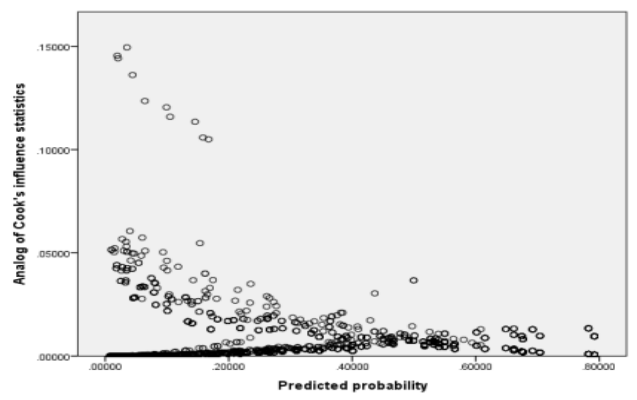

(a)

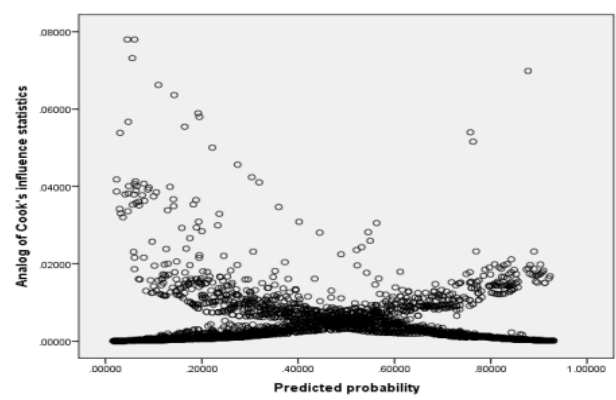

(b)

Fig. 3. Scatterplots for diagnostic checking (Cook's influence statistic), (a) NDHS 2006, (b) NDHS 2011.

Multicollinearity in the constructed model is assessed through the examination of tolerance and Variance Inflation Factors (VIF). VIF's are low for both estimated models (VIF < 1.8). Therefore the models do not suffer from the problem of multicollinearity. Based on these, we may conclude that the model performance is good. Thus, from the above goodness of fit tests and diagnostic checking, we can say that the model is adequate.

\section{Predicted Probabilities for Place of delivery}

Predicting probabilities for delivery at health institute, another logistic regression was fitted with highest education level, wealth index and place of residence as independent variables for both surveys. It is displayed by graph. 

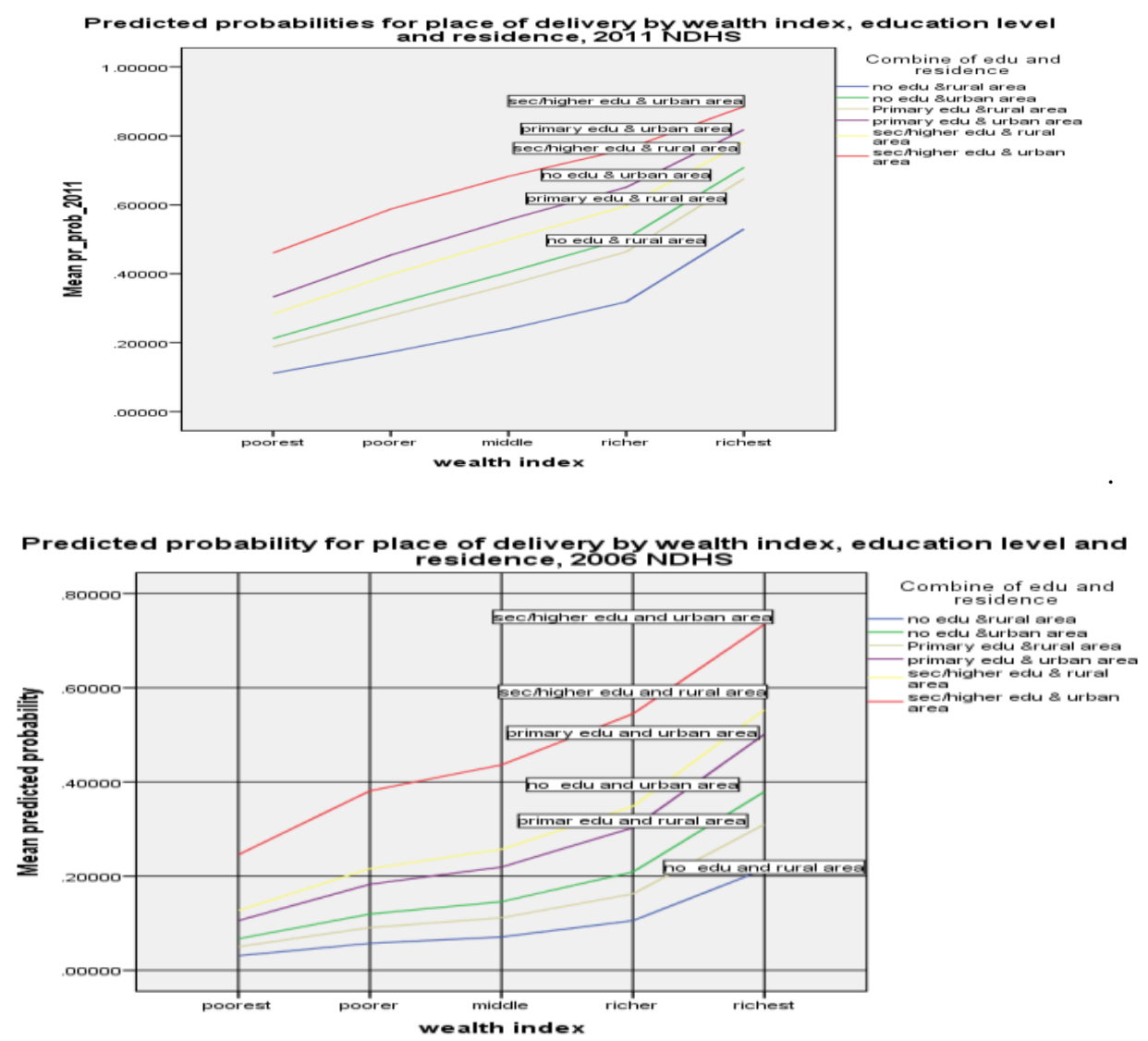

Fig.4. Predicted probability for POD by wealth index, education level and residence (a) NDHS 2006, (b) NDHS 2011.

As display in Figure 4, the probability of delivery at health institute increases as the wealth index increases. However there are differences in the predicted probabilities depending on the education level and place of residence. The predicted probabilities are clustered together towards the lower end of wealth index in 2006. When the wealth index of the household is low, difference in education level and place of residence do not seem to be substantially effect place of delivery. For 2011 NDHS data, the predicted probabilities are not so clustered together towards the lower end of wealth index as in 2006 NDHS data. Figure 4 shows that in all wealth indexes, probability for delivery at health facility for primary education in rural areas is less than for no education in urban areas. Similarly secondary/higher education in rural area is less than primary education in urban areas. It showed that even though less in education level, women from urban areas are more likely to deliver in health institute than women from rural areas. It may be due to the easy access of the health services in urban areas than rural areas. Besides, to get a better understanding of why women do not give birth at a 
health institute, the 2006, 2011 NDHS asked women who gave birth in the five years before the survey, why they didn't give birth in a health institute. Table 5 indicates the reason of not delivery in health institute. In a data set 792 (1615 in 2011 NDHS) respondents delivered at health institute, therefore only 3,390 (2464 in 2011 NDHS) respondents gave the answer of that question.

Table 5. Reason for not delivery at health institute, NDHS 2006 and 2011.

\begin{tabular}{lcccc}
\hline Reason of not delivery in & \multicolumn{2}{c}{2006} & \multicolumn{2}{c}{2011} \\
health facility & Frequency & Percent (\%) & Frequency & Percent (\%) \\
\hline Cost too much & 81 & 2.4 & 42 & 1.7 \\
Facility not open & 11 & 0.3 & 32 & 1.3 \\
Too far/ no transportation & 162 & 4.8 & 314 & 12.7 \\
Don't trust facility & 27 & 0.8 & 34 & 1.4 \\
No female provider & 5 & 0.1 & 6 & 0.2 \\
Husband/ family did & 60 & 1.8 & 53 & 2.2 \\
Not necessary & 2169 & 64.0 & 1396 & 56.7 \\
Not customary & 619 & 18.3 & 256 & 10.4 \\
Security concern & 33 & 1.0 & 7 & 0.3 \\
Child born before & 122 & 3.6 & 208 & 8.4 \\
Others & 101 & 3.0 & 116 & 4.7 \\
\hline Total & 3390 & 100.0 & 2464 & 100 \\
\hline
\end{tabular}

In both survey, lack of awareness appears to be the most important factor for not delivery at health institute (64 percent in 2006 NDHS, 57 percent in 2011 NDHS).

\section{DISCUSSION}

This study investigates the factors that are associated with place of delivery. We now turn to discussion on our findings based on the result of estimation of the logistic regression model. Finally six variables (residence, education level, birth in last five years, wealth index, occupation and ANC by provider) have been found as the most influential variables on place of delivery for 2006 NDHS and 2011 NDHS survey. Age also has been found as the most influential variables on place of delivery for 2011 NDHS survey. It has been shown that increase in education level tended to have increased the use of health institute as place of delivery. Women with secondary/ higher education level are almost 3.2 times (for 2006 NDHS) and 2.3 times (for 2011 NDHS) more to deliver at health institute than odds of women with no education. These results are statistically highly significant. This finding is consistent with many previous studies that showed education of women to be the most 
significant predictor of increased utilization of health services (Bhatia and Cleland, 1995; Becker et al., 1993; Celik and Hotchkiss, 2000; Obermeyer, 1993). There are a number of reasons that education of women has a significant positive relation with maternal health care utilization. Educated women are more likely to realize the benefits of using maternal health services. Therefore, they are more likely to use the services. In addition, education may enhance female autonomy, hence, increasing women's ability to make decisions regarding their own health. Strong association has been found between wealth index and the use of health institute as place of delivery in both survey which is also supported by other studies (Celik and Hotchkiss, 2000; Babalola and Fatusi, 2009). This can be explained by the fact that women should be able to cover the costs needed in order to access health institute. Even in areas where maternal health care services are provided for free, women still have to pay for transportation and additional costs. As a result only those women who can afford to pay for such costs are able to visit health institute.

Interestingly, the relationship between women's occupation and place of delivery has an inverse association. Women whose work is in agricultural sector are 45 percent less likely to give birth at health institute compare to women who never work. The result is statistically significant. Although not statistically significant, it is surprising that women whose occupation is in modern sector are 5 percent and 7 percent less likely to deliver at health institute as compare to women who never work for 2006 NDHS and 2011 NDHS respectively. Women who never work may be associated with household of high economic status and for this reason, an inverse may have been found. Similarly, birth order of the child showed inverse association with use of health institute as place of delivery. Women with birth order 3 or more 58 percent less likely to deliver at health institute as compared to women with $1^{\text {st }}$ birth order for 2006 NDHS. It is statistically significant at 5 percent level of significance. This finding is similar with other studies (Babalola and Fatusi, 2009;). One study from Bangladeshi showed that women with parity of five or more were seen to have a low health seeking behavior when compared to those who had only one child. The possible explanation could be women who have more children usually do not have enough time to go to the health services. In addition to this as the number of children in the household increase there will also be scarcity of resources (Chowdhury, Islam, Gulshan and Chakraborty, 2000).

Urban-rural place of residence served as a control for the study and was used as a proxy for availability and accessibility of health services. Urban rural place of residence emerged significant for place of delivery. The odds ratio suggests that compared to women who lived in rural areas, women who resided in urban areas were about 1.8 times more likely to have delivery in health institute, which is slightly higher based upon 2006 data (OR 1.6). This implies that availability and 
accessibility of health services are important in decisions to seek care. We find that antenatal care is a key determinant of whether a woman gives birth at health institute or at home. In general a woman who goes for antenatal care is more likely to deliver at health institute than a woman who does not go for antenatal checkup. The odds ratio indicates that the women having antenatal care by SBA are likely to have 5.77 times higher to deliver at health institute compared to women having ANC by no one for 2006 NDHS which is lower than 2011 NDHS (OR 7.1). The important finding of this analysis is that in both survey, probability for delivery at health institute for primary education in rural areas is less than for no education in urban areas. Even though less in education level, women from urban areas are more likely to deliver in health institute than women from rural areas. It may be due to the easy access of the health services in urban areas than rural areas (Figure 4).

\section{CONCLUSION}

As noted in the literature review, majority of maternal deaths and disabilities occur around the time of delivery. For this reason, place of delivery was selected as dependent variable. The place of delivery was collapsed to create a dichotomous variable on the basis of whether the woman delivery at health institution or home. A number of independent variables are taken into account based on scientific literature review. In analyzing data, univariate, bi-variate and multivariate analysis were employed. In this study, logistic regression model was used to assess the influence of predictors on place of delivery. Education level, wealth index, birth order, residence and ANC by provider were highly significant predictors for the selection of place of delivery for 2006 NDHS survey but in 2011 NDHS survey age was also highly significant. Residence, education level, wealth index and ANC by provider have a positive association with place of delivery but birth order, occupation and age have a negative association.

Considering model adequacy test such as goodness of fit tests (Hosmer-Lemeshow chi-square statistic), multicollinearity diagnostics (tolerance and VIF), residual analysis and outliers (leverage value) showed that both models fit well to the proposed logistic regression model. Comparing the values of odds ratio obtained from 2006 and 2011 surveys, it is found that the values are only slightly different for most of the predictors under consideration demonstrating consistency of associations found in the two surveys. Few variables like richest wealth index and ANC by SBA have shown marked shifts in their odds ratio between the two surveys.

\section{CONFLICT OF INTEREST}

The author declares that she has no competing interest. 


\section{ACKNOWLEDGEMENTS}

The author is greatly indebted to Professor Dr. Ganga Shrestha for her guidance in this study. The author also expresses gratitude to Professor Dr. Shankar Prasad Khanal and Professor Dr. Srijan Lal Shrestha for their encouragement and valuable suggestions to complete this paper.

\section{REFERENCES}

Adeleke, K., \& Adepoju, A. (2010). Ordinal logistic regression model: An application to pregnancy outcomes. Journal of Mathematics and Statistics, 6 (3), 279-285.

Anderson, R. (1995). Revisiting the behavioral model and access to medical care: Does it Matter. Journal of Health and Social Behavior, 36 (1), 1-10.

Babolola, S., \& Fatusi, A. (2009). Determinant of use of maternal health service in Nigeria, looking beyond individuals and household factors. .BMC Pregnancy and Child Birth, 9, 43.

Becker, S., Peters, D., Gray, R., Gultiano, C., \& Blake, R. (1993).The determinant of Use of maternal and child health services in Metro, Cebu, the Philippines. Health Transition Review, 3, 7789.

Bhatia, J., \& Cleland, J. (1995). Determinants of maternal care in a region of south India. Health Transition Review, 5, 127-142.

Celik, Y., \& Hotchikiss, D. (2002).The socio- economic determinants of maternal health care utilization in Turkey. Social Science and Medicine, 50 (12), 1797-1806.

Chakraborty, N., Islam, M., Chowdhury, R., Bari, W., \& Akhter, H. (2003). Determinant of the use of maternal health Services in rural Bangladesh. Health Promotion International, 18 (4), 327 337.

Chowdhury, R.I., Islam, I.M., Gulshan. J., \& Chakraborty, N. (2000). Delivery complication and health care-seeking behavior: The Bangladesh demographic health survey, 1999-2000. Health and Social Care Community, 15(3), 254-64.

Elo, I. (1992). Utilization of maternal health services in Peru: The role of women's education. Health Transition Review, 2 (1), 49-69.

Gabrysch, S., \& Campbell, O. (2009). Still too far to walk: Literature review of the determinant of delivery services use. BMC Pregnancy and Childbirth, 9, 34.

Gage, A., \& Calixte, M. (2006). Effect of the physical accessibility of maternal health services on their use in rural Haiti. Population Studies, 60 (3), 271-288.

Hosmer, D., \& Lemeshow, S. (2000). Applied Logistic Regression ( $2^{\text {nd }}$ ed.). New York: Wiley. Kroeger, A. (1983). Anthropological and socio medical health care research in developing countries. Social Science and Medicine, 17, 147-161. 
Latemo, G. R. (2003). Factors associated with non use of maternal health services in Botswana. Journal of Health Population and Nutrition, 21 (1), 40-47.

Magadi, M., Madise, N., \& Rodrigues, R. (2000). Frequency and timing of ANC in Kenya: explaining the variation between women of different communities. Social Science and Medicine, 51, 551-556.

Matsumura, M., \& Gubhaju, B. (2001). Women's status, household structure and the utilization of maternal health services In Nepal. Asia-Pacific Population Journal, 16 (1), 23-43.

Ministry of Health [Nepal], New ERA, and ORC Macro. 2002. Nepal Demographic and Health Survey 2001. Calverton, Maryland, Kathmandu, Nepal.

Ministry of Health and Population (MOHP) [Nepal], New ERA, and Macro International Inc. 2007. Nepal Demographic and Health Survey 2006. Kathmandu, Nepal.

Ministry of Health and Population (MOHP) [Nepal], New ERA, and ICF International Inc. 2012. Nepal Demographic and Health Survey 2011. Kathmandu, Nepal.

Nepal Planning Commission. (2002). The Tenth Plan. Kathmandu: Nepal Planning Commission.

Obermeyer, M. (1993). Culture, maternal health care and women's Status: A comparison of Morocco and Tunisia. Studies in Family Planning, 24 (6), 354-365.

Sarin, A. (1997). Under utilization of maternal health services. World Health Forum, 67-68.

Shrestha, G., \& Shrestha G. (2011). Statistical analysis of factors affecting utilization of antenatal care in Nepal. Nepal Journal of Science and Technology, 12, 268-275.

Thind, A., Mohini, A., \& Banerjee, K. (2008). Where to deliver? Analysis of choice of delivery location from a national survey in India. BMC Public Health, 8 (29).

WHO. (1996). Coverage of Prenatal Care: A listing of Available Information. Geneva: World Health Organization.

Reference to this paper should be made as follows:

Shrestha, G. (2017). Factors affecting maternal health care services utilization in Nepal: Insight from the Nepal Demographic Health Survey 2006 and 2011. Nep. J. Stat., 1, 55-72. 